\title{
Competencias de adaptabilidad y factores de éxito académico del alumnado universitario
}

\author{
Pedro-Ricardo Álvarez-Pérez y David López-Aguilar
}

\section{RESUMEN}

En este artículo se analizan los factores que intervienen en la transición, adaptabilidad y éxito en la formación universitaria. Con el estudio se pretendía identificar las características de los estudiantes bien adaptados y con buen rendimiento en la educación superior. Los datos recogidos a través de un cuestionario con una muestra de 208 estudiantes de 36 titulaciones universitarias, reflejaron que el rendimiento en las etapas previas es un factor determinante de la adaptabilidad. Los estudiantes de éxito mostraron metas claras, alta satisfacción con la titulación elegida, buen conocimiento del perfil profesional, constancia en el proceso de estudio, asistencia a clase, etcétera. La fijación de este perfil es importante como referente para la orientación al alumnado preuniversitario, para que desarrollen competencias de adaptabilidad y preparen el acceso a los estudios de grado.

Palabras clave: enseñanza universitaria, éxito académico, competencias de adaptabilidad, abandono académico, España.

Pedro-Ricardo Álvarez-Pérez

palvarez@ull.edu.es

Español. Doctor en Ciencias de la Educación. Profesor Titular de Orientación Profesional en la Universidad de La Laguna, España; Director del Grupo Universitario de Formación y Orientación Integrada (GUFOI); Director del Departamento de Didáctica e Investigación Educativa; Director del Servicio de Orientación e Información al Alumnado y Coordinador del Plan de Tutorías de la Facultad de Educación, Universidad de La Laguna, España (Sitio web: http://palvarez.webs.ull.es). Temas de investigación: transiciones y trayectorias académicas y profesionales del alumnado; abandono, permanencia y éxito en los estudios; orientación y tutoría universitaria. Español. Doctor en Educación. Profesor Ayudante Doctor, Universidad de La Laguna, España; Miembro del grupo de investigación GUFol y del Grupo de Innovación sobre Competencias de Adaptabilidad; Coordinador del área de Investigación, Innovación y Formación del Servicio de Orientación e Información al Alumnado de la Facultad de Educación, Universidad de La Laguna, España (Sitio web: http://dlopez.webs.ull.es). Temas de investigación: procesos de transición académica del alumnado; permanencia en los estudios, y orientación y tutoría universitaria. 


\section{Competências de adaptabilidade e fatores de êxito acadêmico do alunado universitário}

\section{RESUMO}

Neste artigo se analisam os fatores que intervém na transição, adaptabilidade e êxito na formação universitária. Com o estudo se pretendia identificar as características dos estudantes bem adaptados e com bom rendimento na educação superior. Os dados recolhidos através de um questionário com uma amostra de 208 estudantes de 36 cursos universitários, demonstraram que o rendimento nas etapas previas é um fator determinante da adaptabilidade. Os estudantes de êxito mostraram metas claras, alta satisfação com o curso elegido, bom conhecimento do perfil profissional, constância no processo de estudo, presença na aula, etecetera. A fixação deste perfil é importante como referente para a orientação ao alunado pré-universitário, para que desenvolvam competências de adaptabilidade e preparem o acesso ao ensino superior.

Palavras chave: ensino universitário, êxito acadêmico, competências de adaptabilidade, abandono acadêmico, Espanha.

\section{Adaptability skills and factors of academic success among university students}

\section{ABSTRACT}

This article analyzes the factors that intervene in the transition, adaptability and success in university education. The study aimed to identify the characteristics of well adapted and successful students enrolled in higher education. The data collected through a questionnaire with a sample of 208 students from 36 different university degrees showed that performance in the previous stages is a determining factor of adaptability. The successful students manifested clear goals, high satisfaction with the chosen degree, good knowledge of the professional profile, constancy in the study process, class attendance, etc. The establishment of this profile is important as a reference for the orientation of pre-university students, so that they develop adaptability skills and prepare for access to undergraduate studies.

Key words: university education, academic success, adaptability skills, academic dropout, Spain.

Recepción: 24/01/18. Aprobación: 23/09/19. 


\section{Fundamentación teórica}

Un grave problema al que se enfrenta cada año la universidad, es el alto porcentaje de estudiantes de nuevo acceso que tienen problemas para afrontar la transición y adaptarse al nuevo contexto formativo. Efectivamente, la cantidad de estudiantes que inician sus estudios universitarios y presentan un bajo rendimiento académico, que suspenden todas o una parte de las asignaturas y que prolongan o abandonan la formación es considerable en el conjunto de las instituciones de educación superior (IES). Las investigaciones realizadas confirman la relación entre el abandono y variables como la mala articulación entre etapas educativas, las bajas expectativas, la mala toma de decisiones vocacionales, el escaso desarrollo de competencias básicas, la baja autoestima, la inadaptación académica a los nuevos contextos, el bajo rendimiento en los estudios, el escaso compromiso con la carrera, etcétera. (Pascarella y Terenzini, 1991; Álvarez et al., 2006; Elías, 2008; Castaño et al., 2008; Zepke y Leach, 2010; Álvarez et al., 2011; García, 2014; Gonzalo y García, 2016; Himmel, 2018; García-Aretio, 2019). Los resultados de las investigaciones han reflejado también que el fracaso en los estudios y la decisión de abandonar la formación se ve condicionada por múltiples factores. Como indica Rué (2013: 283), es necesario entender la deserción como un problema "complejo y generado tanto por factores exógenos a la formación como endógenos, así como una compleja relación entre ambos en los distintos contextos en los que se registra".

El abandono en los contextos educativos tiene una enorme trascendencia a nivel mundial, puesto que como señalan Tejedor et al. (2007: 443), "el bajo rendimiento académico, el excesivo tiempo invertido en el estudio de una titulación, el abandono de los estudios, son problemas comunes a todos los países de nuestro entorno cultural y económico". La situación se ha complejizado en los últimos tiempos, dado que el perfil de los estudiantes que llegan a la universidad no es homogéneo, lo que hace más difícil la adaptación. Actualmente acceden a los estudios universitarios, no sólo los que proceden de la secundaria y el bachillerato, sino también alumnado de otros países, estudiantes que habían abandonado la formación y se reincorporan, trabajadores que buscan actualizar sus conocimientos, etcétera.

Todo esto ha generado un marco de condiciones complejo y ha evidenciado también las dificultades que tiene la universidad para responder de manera eficiente al abandono académico, problema que se vincula directamente con criterios de calidad institucional. La "tasa de deserción" (proporción de estudiantes matriculados que por diversos motivos abandonan sus estudios sin haberlos completado), constituye un potente indicador de calidad en diferentes modelos de evaluación de la educación superior y se emplea también como indicador para elaborar los rankings de universidades (Cabrera et al., 2006). Aunque parezca un contrasentido, muchas veces las instituciones universitarias están más preocupadas por la competencia, el marketing y la captación de estudiantes, que por diseñar políticas y programas para apoyar y asesorar a los estudiantes con la finalidad de que se integren bien y alcancen el éxito en sus estudios.

A nadie escapa que los altos porcentajes de abandono o prolongación de los estudios que se vienen registrando en la Universidad en general y, en algunas titulaciones en particular, constituyen un indicador de calidad negativo. Por el contrario, lograr que el alumnado se integre y tenga éxito en los estudios, se considera un indicador de calidad positivo para la institución y una fortaleza para poder cumplir con los objetivos de la universidad: formar jóvenes bien cualificados que puedan insertarse en la sociedad y en el mundo del trabajo.

En el análisis del problema se ha detectado que la preparación de la transición, las expectativas hacia la formación universitaria, la calidad de los recursos que ofrece la institución para que el estudiante pueda realizar su trabajo académico, el clima social y los 
apoyos recibidos, son factores importantes que intervienen en las posibilidades de integración y éxito en la educación superior (Feldman, 2005; Tinto, 2006; Villar, 2010; Nora y Crips, 2012; Álvarez y López, 2017). Muchos estudiantes que acceden a la universidad no tienen conocimiento de la metodología de trabajo, no poseen las competencias adecuadas para adaptarse a los nuevos estudios, no tienen habilidades de planificación y gestión del proceso de estudio, no tienen una hoja de ruta para su itinerario formativo y no tienen autonomía en el proceso de aprendizaje.

El estudio realizado por Martín et al. (2010) en el ámbito de la educación superior, puso de manifiesto que un porcentaje alto de estudiantes, sólo cuando llegan a la universidad se dan cuenta de que se han equivocado de titulación, que no poseen las capacidades adecuadas para abordar las tareas académicas y que los estudios que han elegido no les satisfacen. Estaríamos ante una cohorte de estudiantes cuya preparación y formación para afrontar la transición y adaptación a la formación universitaria es inapropiada, dado que ni poseen la información adecuada, ni han desarrollado las habilidades para hacer frente a estas situaciones que exigen autonomía, capacidad de decisión, capacidad de adaptarse a nuevas situaciones, etcétera. Sousa, Lopes y Ferreira (2013: 404) destacan que la entrada a la educación superior es un momento crítico en las trayectorias formativas, que requiere la puesta en acción de "un conjunto de estrategias por parte de cada estudiante para readaptarse, ya que se enfrentará a un conjunto de alteraciones significativas: cambio de espacio institucional, prácticas pedagógicas, tareas académicas, mayor nivel de responsabilidad y autonomía". La investigación realizada por Pérez, Quijano y Muñoz (2018: 167) mostró el estado de incertidumbre e indecisión de muchos estudiantes en relación con su integración a la universidad, en aspectos claves como "la autoestima y autoconcepto, de modo que hay que mejorar la acogida inicial, para favorecen la seguridad personal".
No se puede obviar que los periodos de transición se han diversificado y que la adaptación a la formación universitaria depende de muchos factores psicopedagógicos. Los estudios realizados ratifican el fuerte peso que tiene en la integración, en el rendimiento en las materias de primer curso universitario y en el éxito académico del alumnado la formación previa, la capacidad de relacionarse y hacer nuevas amistades en el contexto universitario y el domino de competencias de adaptabilidad diversas que facilitan la acomodación a diferentes contextos (Rodríguez et al., 2004; Bethencourt et al., 2008; Belvis et al., 2009; Jansen et al., 2010; Álvarez, López y Pérez, 2015; García et al., 2016; Sánchez y Elías, 2017; Pérez, Quijano y Muñoz, 2018). Para Ramírez, Herrera y Herrera (2003), la adaptabilidad es una capacidad intelectual y emocional para responder de manera coherente a las exigencias del entorno, a través de un proceso dinámico de ajuste y regulación en función de las características del contexto. Para Savickas (2012) la adaptabilidad de carrera es una dimensión de la adaptabilidad profesional, que contempla cuatro dimensiones (implicación, control, curiosidad y confianza) cuyo desarrollo requiere el domino de distintas estrategias de autorregulación en la persona. En los contextos educativos, Granada (2003) plantea que cuando se produce una interacción adecuada entre la persona y el entorno, se incrementan las posibilidades de persistencia, de bienestar en el alumno y de logro de los objetivos.

En el estudio realizado por González et al. (2007) se concluyó que la persistencia para acabar la titulación a pesar de los obstáculos; el interés y motivación de los estudiantes hacia la titulación elegida; la satisfacción con los estudios cursados; las expectativas favorables de resultados, y el ajuste entre las capacidades del sujeto y las exigencias de la formación, son garantías de éxito académico. El modelo sociocognitivo del desarrollo de la carrera (Lent y Hackett, 1987; Lent et al., 2004) destaca que las expectativas de autoeficacia y el componente social son 
dos factores claves que actúan directamente sobre las posibilidades de integración académica y éxito en los estudios (Lent et al., 2009). La autoeficacia constituye actualmente un referente fundamental para abordar el análisis de las variables asociadas a la integración en diferentes contextos y tramos del sistema educativo. En la investigación llevada a cabo por Honike y Broadbent (2016) se analizó la compleja relación entre la autoeficacia académica y el rendimiento académico. Identificaron distintos elementos mediadores entre ambos factores, como la regulación de esfuerzo, las estrategias de procesamiento de la información y las orientaciones de objetivos. Yokoyama (2018) estudió la relación entre la autoeficacia académica y rendimiento en procesos de aprendizaje en línea. Concluyó que aquellos estudiantes que no poseen buenas habilidades en el uso de dispositivos de aprendizaje en línea tienen más dificultades para lograr el éxito académico. Por ello, la autoeficacia y las expectativas favorables de resultados académicos, se consideran un buen predictor de la adaptabilidad a un área académica o titulación determinada, dado que las personas eligen actividades y toman decisiones vocacionales a partir de sus mecanismos cognitivos de evaluación de competencias.

De acuerdo con esta perspectiva, en este estudio se pretende clarificar los rasgos que definen el perfil de un estudiante universitario de éxito. Concretamente, se busca identificar las características psicosociales y psicopedagógicas que tienen los estudiantes que se adaptan bien y tienen buen rendimiento académico en los estudios universitarios. Esta información puede ser de gran relevancia para que en las etapas previas se oriente y se trabaje el desarrollo de competencias de adaptabilidad de cara a la posterior integración social y académica del alumnado a la educación superior. En relación con este planteamiento, los objetivos específicos propuestos para el estudio son:

- Analizar las expectativas del alumnado respecto a los estudios universitarios.

- Valorar los factores que tiene en cuenta el alumnado al elegir estudios universitarios.

- Analizar los factores relacionados con el rendimiento académico.

- Conocer qué competencias y estrategias manejan los estudiantes de éxito.

- Conocer las expectativas en relación con su futuro profesional.

\section{Método}

\section{Participantes}

Para la selección de los participantes, se aplicó un procedimiento de muestreo causal o intencional con la finalidad de identificar estudiantes que reunieran las siguientes características:

- Estudiantes de segundo, tercero o cuarto curso de grado universitario que tuvieran, en el momento de recogida de los datos, un rendimiento académico medio superior a 8.5 (notable).

- Estudiantes que hubieran superado la evaluación la primera vez que se presentaron a una convocatoria en cada asignatura.

Aplicando estos criterios, se identificó un total de 689 estudiantes de la Universidad de La Laguna (España) que cumplían con los requisitos establecidos $(\mathrm{N}=689)$. Finalmente, se seleccionaron de manera aleatoria 208 estudiantes $(n=208)$ de 36 titulaciones de grado, pertenecientes a diferentes facultades y escuelas, lo que permitió trabajar con un nivel de confianza del $95.0 \%$ y un margen de error de $\pm 5.7 \%$. 
Tabla 1. Características generales de la muestra participante en el estudio

\begin{tabular}{|c|c|c|c|}
\hline Sexo & Edad & Rama de conocimiento & Curso \\
\hline Hombres $=43.3 \%(n=90)$ & Rango $=18-57$ & Ciencias Sociales y Jurídicas $=24.0 \%(n=50)$ & $2^{\circ}=32.2 \%(n=67)$ \\
\hline Mujeres $=56.7 \%(118)$ & Media $=22.82$ & Artes y Humanidades $=23.6 \%(n=49)$ & $3^{\circ}=24.5 \%(n=53)$ \\
\hline 3 & Mediana $=21.0$ & Ciencias $=17.3 \%(n=36)$ & $4^{\circ}=43.3 \%(n=90)$ \\
\hline & $\mathrm{dt}=6.3$ & Ciencias de la Salud $=27.4 \%(n=57)$ & \\
\cline { 2 - 4 } & & Ingenierías y Arquitectura $=7.7 \%(n=16)$ & \\
\hline
\end{tabular}

Fuente: elaboración propia.

Las titulaciones en las que hubo mayor número de participantes fueron los grados de Medicina (8.2\%), Psicología (7.7\%), Enfermería (5.5\%), Física (4.8\%), Biología $(4.3 \%)$ y Derecho $(4.3 \%)$. En cuanto a la distribución por género, el $43.3 \%$ fueron hombres y el 57.6\% mujeres. La edad media de los participantes fue de 22.8 años, con una desviación típica de 6.3. La vía de acceso a los estudios universitarios fue mayoritariamente la Prueba de Acceso a la Universidad $(89.4 \%)$ o la prueba para mayores de 25 años $(2.4 \%)$.

\section{Instrumento de recogida de información}

Para la recogida de datos se utilizó el "Cuestionario sobre Factores de Exito del Alumnado Universitario (CUFE)". Se trata de un instrumento elaborado ad hoc y adaptado a los objetivos del estudio y a las características específicas de la muestra a la que iba dirigido. En concreto, el cuestionario se diseñó a partir de los presupuestos teóricos que dieron soporte justificativo a las dimensiones que se pretendían analizar a través del trabajo de campo. Durante el proceso de construcción de esta prueba, se realizaron diferentes procedimientos de validez propuestos por McMillan y Schumacher (2005), con la finalidad de garantizar su adecuación para evaluar las dimensiones y variables del estudio. Concretamente se realizaron las siguientes pruebas:

- Prueba piloto: en la que participaron veinte estudiantes que reunían características similares a las de la muestra definitiva. A esta prueba asistieron dos investigadores con la intención de anotar aquellas posibles incidencias relativas al diseño del cuestionario. Con esta prueba se midieron los tiempos de respuesta, la claridad y comprensión de los ítems, las alternativas de respuesta, etcétera.

- Prueba de contenido: esta prueba fue realizada por tres profesores universitarios especialistas en el ámbito a de la orientación académica. Los criterios de valoración que se tuvieron en cuenta en esta prueba fueron la pertinencia, claridad, interés y adecuación de las preguntas a los objetivos de la investigación.

- Prueba de forma: dos profesores expertos en el ámbito de la metodología de la investigación educativa llevaron a cabo esta prueba con la intención de valorar los tipos de preguntas y escalas a utilizar, los posibles análisis estadísticos a realizar, el número de ítems, etcétera.

Estas pruebas permitieron depurar y definir el cuestionario definitivo que fue aplicado a la muestra objeto de estudio. Tras introducir las correcciones oportunas a partir de las pruebas realizadas, se diseñó la versión definitiva del cuestionario, que quedó finalmente conformada por un total de 26 preguntas de diferente tipo: preguntas cerradas con escalas de valoración de 6 niveles (escala tipo Likert, donde 1 hacía referencia a nada de acuerdo y 6 a totalmente de acuerdo; la distancia existente entre las distintas 
alternativas de respuesta de las escalas de valoración utilizadas se consideraron iguales a fin de llevar a cabo análisis de contraste) preguntas dicotómicas (Sí - No) y preguntas abiertas.

En cuanto a la validez de criterio del cuestionario empleado, se obtuvo mediante el paquete de estadística Statistical Package for the Social Sciences (SPSS) versión 17.0 para el entorno Microsoft Windows 7. El índice general obtenido para los 67 elementos que conformaban las escalas tipo Likert utilizadas en la prueba fue de $a=0.901$, lo que indicó que existía una alta consistencia interna y un nivel de estabilidad de la prueba significativo (George y Mallery, 2003).

\section{Procedimiento y análisis de datos}

El procedimiento de administración de la prueba se llevó a cabo durante el último semestre del curso 2014-2015, mediante el envío de un cuestionario online a través de correo electrónico a la muestra de estudiantes participantes, acompañado de una carta en la que se explicaban las finalidades del estudio, así como el hipervínculo al que debían remitir el instrumento de recogida de datos una vez cumplimentado. Se seleccionó la herramienta de encuestas online LimeSurvey, ya que se adaptaba a los objetivos planteados, ofrecía la posibilidad de almacenar un número ilimitado de respuestas, permitía introducir una amplia variedad de formatos de preguntas y, además, facilitaba la elaboración de bases de datos autoexportables a programas estadísticos como el SPSS o el Excel.

Una vez recogida la información a través del cuestionario online, los datos fueron tratados estadísticamente empleando el software informático SPSS 17.0 para el sistema operativo Microsoft Windowes 7. De manera más específica, los exámenes estadísticos realizados fueron:

- Análisis de fiabilidad a los ítems de valoración del cuestionario mediante la prueba Alfa de Cronbach.
- Análisis descriptivos para identificar las medidas de tendencia central y la distribución de frecuencias relacionadas con las dimensiones y variables estudiadas con el cuestionario (Etxeberría y Tejedor, 2005).

- Análisis comparativo entre variables con la finalidad de identificar posibles diferencias estadísticamente significativas en la muestra objeto de estudio. Las pruebas empleadas fueron y análisis de la varianza (ANOVA). Como paso previo, se realizaron pruebas sólidas de igualdad de medias a través de los estadísticos de Welch y Brown-Forsythe con la finalidad de comprobar los supuestos de semejanza de la varianza de las escalas empleadas.

- Tamaño del efecto para calcular la magnitud de las diferencias encontradas. Para ello se utilizó el estadístico $\hat{\mathrm{g}}$ de Hedges para los contrastes realizados con $t$-student y eta cuadrado $\left(\eta^{2}\right)$ para los realizados mediante ANOVA. Los valores del tamaño del efecto de la $\hat{\mathrm{g}}$ de Hedges fueron calculados en el software informático Microsoft Excel 2013 mediante la siguiente fórmula:

$$
\hat{\mathrm{g}}=\frac{\overline{\mathrm{x}_{1}}-\overline{\mathrm{x}_{2}}}{\sqrt{\frac{\left(\mathrm{n}_{1}-1\right) \mathrm{s}_{1}^{2}+\left(\mathrm{n}_{2-1}\right) \mathrm{s}_{2}^{2}}{\mathrm{~N}-2}}} \times\left(1-\frac{3}{4\left(\mathrm{n}_{1}+\mathrm{n}_{2}\right)-9}\right)
$$

Para la interpretación de los resultados del tamaño del efecto, se tuvieron en cuenta los valores aportados por Cohen (1969) y Coe y Merino (2003).

- Análisis de contenido para valorar las respuestas ofrecidas por los estudiantes en las preguntas abiertas del cuestionario (López, 2002). Las aportaciones de cada uno de los participantes fueron identificadas con la codificación $P n$ (donde $P$ hacía referencia a la palabra "participante" y $n$ representaba el "número" de encuestado). 


\section{Resultados}

Los resultados de la investigación se presentan agrupados en función de las diferentes dimensiones y variables relacionadas con los objetivos formulados.

\section{Expectativas y elección de estudios universitarios}

En cuanto al momento en el que habían elegido la titulación universitaria que estaban cursando (gráfica 1), la mayoría había tomado la decisión desde la etapa de bachillerato (31.7\%), tras finalizar la Prueba de Acceso a la Universidad (PAU) (18.3\%) o a lo largo de su formación en la Educación Secundaria Obligatoria (ESO) (13.5\%). Por otra parte, y en menor medida, un grupo de estudiantes decidió cursar los estudios en los que estaba matriculado mientras se encontraba en activo en el mercado laboral $(12.0 \%)$ o cuando estaba cursando estudios de Formación Profesional (FP) (3.4\%). Destaca también en este sentido, que un $13.9 \%$ señaló que desde siempre había tenido claro los estudios universitarios que quería realizar.

\section{Gráfica 1. Momento de la elección de los estudios universitarios}

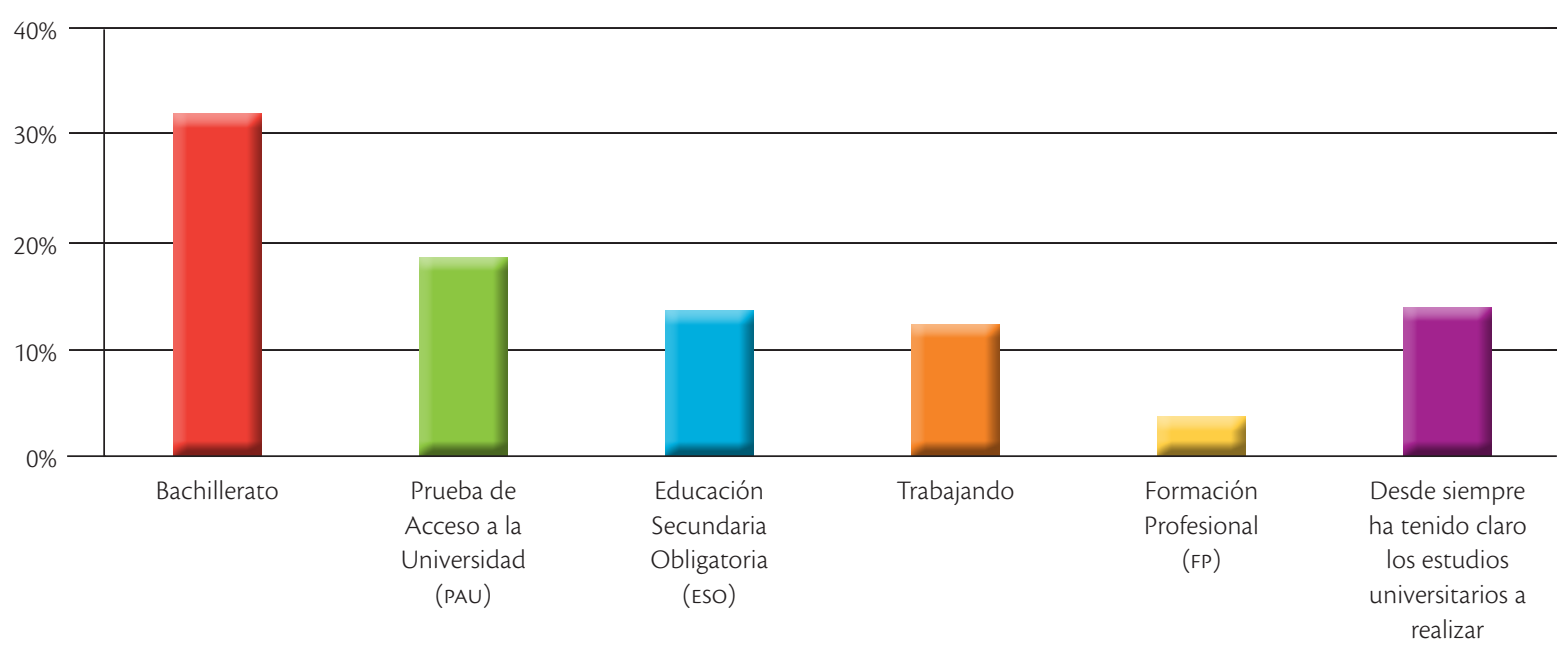

Fuente: elaboración propia.

Los datos revelaron que el $62.5 \%$ de los estudiantes se preocuparon por obtener buenas calificaciones durante la etapa de bachillerato, con la finalidad de obtener plaza en la titulación universitaria que deseaban cursar. En este sentido, destacó que para el 31.7\% las notas de bachillerato fueran determinantes en la elección de los estudios universitarios que estaban cursando.

Como se aprecia en la tabla 2, los factores que mayor peso tuvieron para los estudiantes universitarios de éxito a la hora de elegir titulación universitaria fueron: la preferencia o gusto por la titulación, el ajuste a las características y capacidades personales y las salidas profesionales que le ofrecía el título universitario. En un análisis más preciso de esta información, se constató que el alumnado perteneciente a la rama de conocimiento de ciencias de la salud eligió su formación universitaria motivado principalmente por las salidas profesionales que le ofrecía $\left(\bar{x}=4.51 ; \mathrm{p} \leq 0.000 ; \mathrm{F}=7.588 ; \mathrm{\eta}^{2}=0.130\right)$ y por el prestigio social que tenían las titulaciones relacionadas con el área médica $(\bar{x}=2.77 ; \mathrm{p} \geq 0.000 ; \mathrm{F}=5.640$; $\left.\eta^{2}=0.100\right)$. Por su parte, el grupo de estudiantes de la rama de ciencias, eligieron este tipo de estudios universitarios por la preferencia o gusto por la carrera $(\bar{x}$ $\left.=5.75 ; \mathrm{p}=0.020 ; \mathrm{F}=2.989 ; \eta^{2}=0.056\right)$. 
Tabla 2. Factores que intervienen en la elección de estudios universitarios

\begin{tabular}{l|c|c|c|c|c|c|c|c} 
& $\mathbf{1}$ & $\mathbf{2}$ & $\mathbf{3}$ & $\mathbf{4}$ & $\mathbf{5}$ & $\mathbf{6}$ & $\overline{\boldsymbol{X}}$ & $\boldsymbol{d t}$ \\
\hline Prestigio social de la carrera & 91 & 41 & 38 & 23 & 8 & 7 & 2.22 & 1.382 \\
\hline Preferencia o gusto por la carrera & 1 & 2 & 4 & 21 & 36 & 144 & 5.50 & 0.885 \\
\hline Salidas profesionales de los estudios & 20 & 23 & 31 & 54 & 50 & 30 & 3.87 & 1.506 \\
\hline Exigencias de mis padres & 173 & 20 & 8 & 3 & 2 & 2 & 1.30 & 0.828 \\
\hline No poder acceder a otra carrera por la nota & 191 & 5 & 4 & 5 & 0 & 3 & 1.21 & 0.799 \\
\hline Facilidad de esta carrera & 162 & 25 & 11 & 4 & 3 & 3 & 1.41 & 0.964 \\
\hline Es una carrera que se ajusta a mis características & 4 & 3 & 22 & 28 & 75 & 76 & 4.90 & 1.165 \\
\hline
\end{tabular}

Fuente: elaboración propia.

Por otra parte, llamó la atención el poco peso que tuvieron algunos factores como la facilidad de la carrera o las exigencias de los padres de los estudiantes a la hora de seleccionar un título universitario. De cualquier manera, destacó la alta motivación y la alta satisfacción que tenían los estudiantes universitarios respecto a la titulación que estaban cursando (en cuanto a los contenidos, resultados obtenidos, relación con los compañeros, etcetera).

La alta satisfacción con los estudios que estaban cursando explica que el $81.7 \%$ de los estudiantes universitarios de éxito no se hubieran planteado cambiar de titulación o abandonar la formación universitaria, dado que (participante $8, \mathrm{p} 8$ ) "la carrera que curso me gusta y se ajusta a mis capacidades y competencias", (p14) "me siento cómoda y feliz con lo que curso actualmente", (p23) "me encanta mi carrera; me llena y me siento afortunado de estar estudiándola" o (p87) "es exactamente la disciplina que me interesa estudiar y en la que quiero especializarme profesionalmente".

Otro de los aspectos que reveló la investigación, fue la reconsideración que algunos estudiantes realizaron de la elección de estudios universitarios, con base en nuevas informaciones o circunstancias personales. Concretamente, señalaron que (p160) "mi primera opción era el grado de Medicina y tenía la nota para entrar (...) sin embargo, tras realizar las prácticas del Ciclo Formativo de Grado Superior en el Hospital y tras asistir a las charlas sobre las distintas carreras, cambié de decisión hacia una carrera que se adaptaba más y de manera más realista a mis intereses" o (p139) "al acabar bachillerato, mi primera opción fue Física, pero fracasé en esa carrera, hice otras cosas y tres años más tarde volví a la universidad con muchas ganas de hacer Psicología, que había sido mi segunda opción".

Asimismo, algunos encuestados indicaron que habían cursado más de una titulación o que habían decidido cursar otro grado para completar su formación académica. En las respuestas a las preguntas abiertas del cuestionario, los estudiantes constataron que (p127) "me titulé primero de otra titulación y quise seguir con mi formación profesional”, (p158) "terminé una licenciatura y decidí empezar un grado" y (p181) "poseo estudios previos en el ámbito del Diseño".

En cuanto a la información sobre la titulación universitaria que estaban cursando, el $82.7 \%$ indicó que conocía con precisión el perfil profesional de los estudios. Aquellos que manifestaron no conocerlo 
(17.3\%), señalaron que era debido a que (p87) "conozco una parte, la más conocida en general, pero no en profundidad por falta de información", (p159) "porque según el ámbito de especialización el perfil varía, por lo que no puedo decir que lo conozca con precisión solo a nivel general" o porque (p90) "tiene muchos perfiles, pero nadie informa hasta que llegas a tercero".

Como se aprecia en la tabla 3, los participantes consideraron que, a la hora de tomar una decisión importante, había que tener en cuenta las consecuencias de la decisión a tomar, elegir la alternativa más conveniente después de reunir abundante información sobre las opciones disponibles, decantarse por la alternativa que se ajustara mejor a las capacidades y habilidades personales y priorizar las alternativas a las que podía acceder.

De manera mayoritaria, a la hora de tomar decisiones trascedentes, los participantes manifestaron no dejarse llevar por las opiniones de los demás y no elegir sin pararse a reflexionar y valorar las diferentes opciones. En este sentido, los datos reflejaron la importancia de no dejarse influenciar, puesto que ello podría conducir a situaciones desfavorables y poco satisfactorias. En definitiva, en los procesos de toma de decisiones que podían incidir directamente en el rumbo de la vida de cada persona, los estudiantes universitarios de éxito consideraron que había que valorar los criterios personales y aislarse de la influencia de factores externos.

Tabla 3. Criterios para la toma de decisiones académicas y/o profesionales

\begin{tabular}{|c|c|c|c|c|c|c|c|c|}
\hline & 1 & 2 & 3 & 4 & 5 & 6 & $\bar{x}$ & $d t$ \\
\hline Te dejas llevar por las opiniones y consejos de los demás & 58 & 84 & 54 & 11 & 1 & 0 & 2.10 & 0.887 \\
\hline Valoras las consecuencias de la decisión que vas a tomar & 0 & 3 & 16 & 35 & 65 & 89 & 5.06 & 1.017 \\
\hline Eliges cualquier opción sin pararte a valorar las demás & 145 & 46 & 12 & 1 & 1 & 3 & 1.44 & 0.861 \\
\hline Priorizas las alternativas a las que puedes optar & 5 & 17 & 45 & 52 & 50 & 39 & 4.16 & 1.316 \\
\hline $\begin{array}{l}\text { Buscas información sobre las distintas opciones y eliges la que consideras más } \\
\text { conveniente }\end{array}$ & 3 & 5 & 19 & 37 & 72 & 72 & 4.86 & 1.150 \\
\hline Eliges aquella opción que mejor se ajusta a tus capacidades y habilidades & 1 & 5 & 21 & 30 & 70 & 81 & 4.95 & 1.111 \\
\hline
\end{tabular}

Fuente: elaboración propia.

\section{Experiencia y resultados de aprendizaje en la etapa preuniversitaria}

En relación con el rendimiento académico que el alumnado participante en el estudio había tenido durante la etapa de secundaria obligatoria (ESO) y el bachillerato, tan sólo el 2.9\% había repetido algún curso (un 1.9\% lo había hecho en primero de bachillerato y un $1.0 \%$ en segundo). Algunos motivos que apuntaron los estudiantes para explicar esta situación fueron porque (p73) "cambié de instituto y de contexto social", (p190) "cambié de instituto y no estuve centrada durante un tiempo" o (p171) "repetí GOU porque estuve poco motivada ese curso".

En cuanto a la nota media que obtuvieron en la etapa de bachillerato (gráfica 2), el 29.1\% alcanzó la calificación de notable, el 27.2\% de sobresaliente y el 38.8\% de matrícula de honor. Tan solo el 4,9\% había finalizado sus estudios preuniversitarios con un bien (6 sobre 10). De la misma manera, en el momento de realizar el estudio, la nota media global del alumnado participante fue de notable $(63.0 \%)$ y sobresaliente $(37.0 \%)$. 


\section{Gráfica 2. Calificaciones obtenidas durante la etapa preuniversitaria}

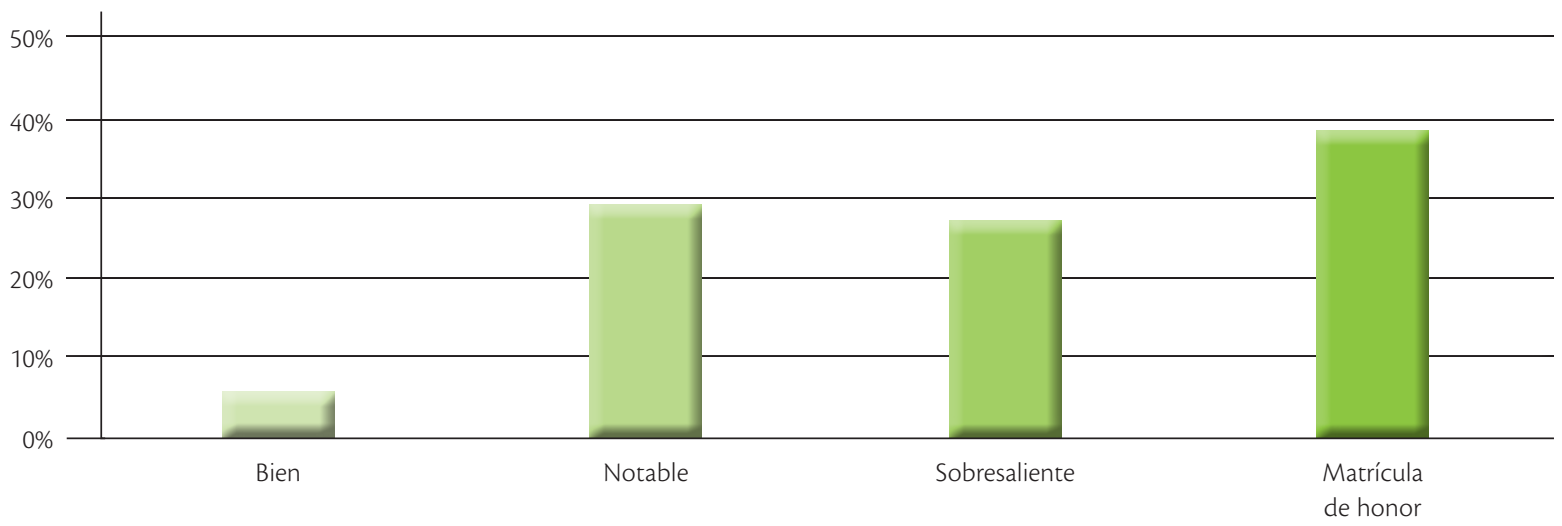

Fuente: elaboración propia

\section{Proceso de estudio, estrategias y rendimiento académico en la universidad}

El 33.2\% del alumnado encuestado señaló que habían encontrado algunas dificultades en su proceso formativo universitario. Concretamente, hacían referencia a (p3) "dificultades de entendimiento debido a la mala explicación del profesorado", (p27) "dificultad con los contenidos de algunas asignaturas y para ser capaz de realizar simultáneamente gran cantidad de trabajos", (p159) "problemas para sintetizar la información y las fuentes bibliográficas que se ofrecen, realizar los trabajos académicos con tiempo de disfrutarlos y relacionar contenidos evitando estudiar de memoria" o (p198) "problemas de organización porque tenemos el tiempo justo para estudiar todas las asignaturas".

Como media, los estudiantes dedicaron al estudio 22.13 horas semanales $(\overline{\boldsymbol{x}}=22.13$; sd $=36.24)$. De manera más precisa (gráfica 3), algunos alumnos estudiaban todos los días a lo largo del año $(37.5 \%)$, otros lo hacían los días próximos a exámenes $(27.9 \%)$ y finalmente se encontró un grupo que solo dedicaba algunos días de la semana al estudio $(26.9 \%)$. En relación con la frecuencia del proceso de estudio, los estudiantes consideraron que (p22) "el secreto está en una buena organización y yo dedico los cinco días laborales de la semana al estudio principalmente", (p47) "soy consciente de que hay algunas asignaturas que no las hubiese sacado si no hubiera estado constantemente con ellas, por lo menos Derecho".

Según los datos obtenidos en la investigación, uno de los factores más determinantes para lograr un buen rendimiento académico en la universidad era la asistencia regular a clase. De hecho, las pruebas de $t$-student revelaron que los estudiantes que alcanzaron calificaciones de sobresaliente en la enseñanza universitaria eran aquellos que se preocuparon por acudir a diario al aula $(\overline{\boldsymbol{x}}=5.90 ; \mathrm{p}=0.011 ; \mathrm{F}=17.432$; $\hat{\mathrm{g}}=0.33)$. Efectivamente, el $93.3 \%$ de los estudiantes indicó que asistía regularmente a las clases de todas las asignaturas en las que estaba matriculado. Entendían que se trataba de una estrategia básica para tener éxito en los estudios superiores, ya que (pl) "es sumamente importante coger apuntes adecuados a tu estilo de estudiar, aparte que siempre puedes preguntar cualquier cosa que no hayas entendido y escuchar las explicaciones de los profesores", (p24) "me resulta más fácil afrontar el estudio de un temario que alguien me ha explicado antes, en lugar de enfrentarme a él desde cero [...] me ayuda a mantenerme motivada $[\ldots]$ me parece extremadamente tediosa la idea de estudiarme todo el temario por mi 
cuenta, sin haber ido a clase, sin interactuar con personas que están estudiando lo mismo que yo [...]; por último pero no menos importante, adoro mi carrera $[. .$.$] no asisto a clase porque sea una obligación$ o sólo para aprobar, sino porque la mayoría de ellas las disfruto y me aportan algo a nivel personal" o (p159) "esas horas las considero de estudio y el desarrollo de unos apuntes propios y la posibilidad de hacer preguntas directas en clase me parece crucial para superar las asignaturas".

\section{Gráfica 3. Tiempo de dedicación al estudio}

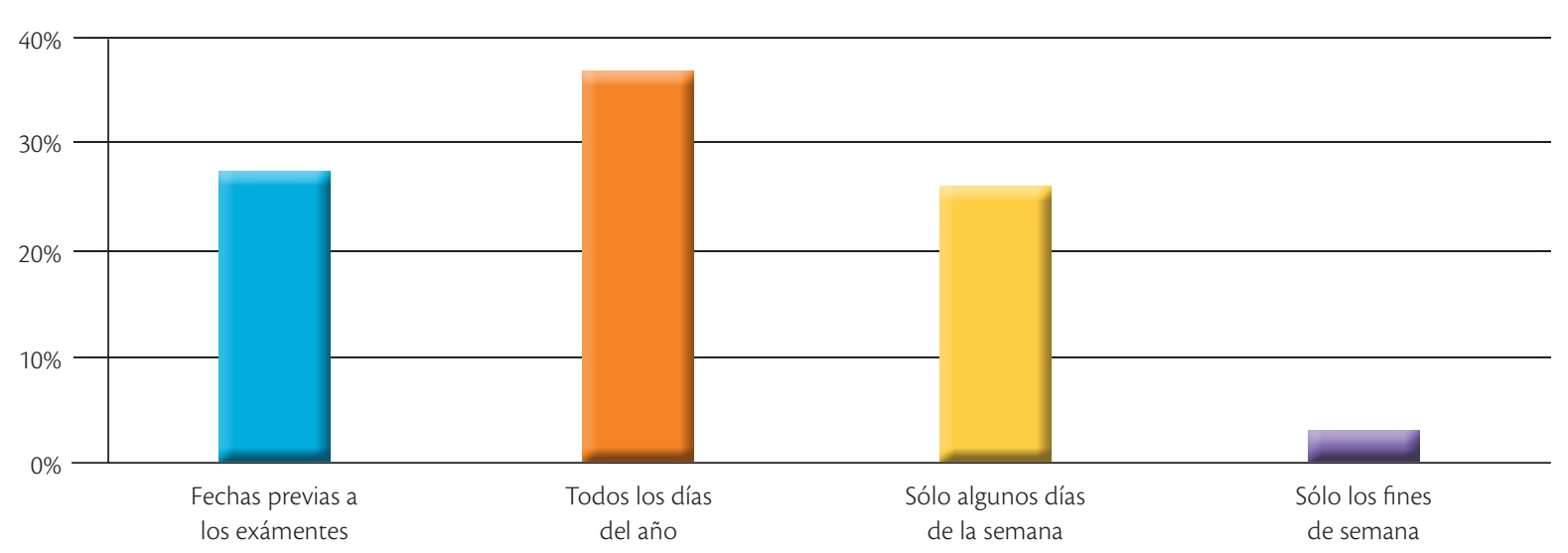

Fuente: elaboración propia.

El grupo de estudiantes que no podía asistir regularmente a clase $(6.7 \%)$, señaló que para mantenerse al día en su proceso de aprendizaje (p28) "consultó apuntes de otros años, el material proporcionado por el profesor y libros", (p112) "consigo los apuntes y estudio por mi cuenta" o (p137) "me encargo de pedir los apuntes dados; me preparo también con el material facilitado en el campus virtual y la guía docente".

En cuanto a la asistencia a las tutorías académicas, el 43.3\% de los encuestados indicó que acudía con frecuencia para hablar con el profesor, con el fin de (p1) "maximizar mi entendimiento conforme a lo que me piden en las asignaturas", (p13) "preguntar y aclarar dudas", (p109) "mejorar el trabajo realizado durante el cuatrimestre" o (p147) "asentar conocimiento y preguntar dudas sobre conceptos, ejercicios mandados". Quienes no asistían a las tutorías académicas $(56.7 \%)$ señalaron que se debía a que (p10) "me es difícil asistir por cuestión de horarios", (p24) "como asisto a clase regularmente, aprovecho para resolver allí las dudas que se me plantean", (p135) "acudo sólo cuando me pierdo alguna clase o tengo alguna duda muy urgente" o (p205) "no son espacios formativos para nada, echo de menos que sea un espacio para el aprendizaje más en profundidad de la materia".

Respecto a los sistemas de evaluación y como se puede apreciar en la tabla 4, el tipo de pruebas con las que obtuvieron mejores resultados los estudiantes de éxito encuestados, fueron las tareas individuales, los exámenes de preguntas de desarrollo, los exámenes de preguntas cortas, la resolución de casos prácticos o los trabajos en equipo. 
Tabla 4. Preferencia sobre los tipos de evaluación

\begin{tabular}{l|c|c|c|c|c|c|c|c} 
& $\mathbf{1}$ & $\mathbf{2}$ & $\mathbf{3}$ & $\mathbf{4}$ & $\mathbf{5}$ & $\mathbf{6}$ & $\overline{\boldsymbol{X}}$ & $\mathbf{d t}$ \\
\hline Examen de preguntas cortas & 3 & 11 & 29 & 49 & 73 & 43 & 4.48 & 1.200 \\
\hline Examen de preguntas de desarrollo & 3 & 4 & 17 & 42 & 67 & 75 & 4.88 & 1.134 \\
\hline Examen tipo test & 12 & 27 & 32 & 50 & 48 & 39 & 4.02 & 1.478 \\
\hline Examen oral & 20 & 32 & 43 & 41 & 46 & 26 & 3.67 & 1.520 \\
\hline Resolución de casos prácticos & 0 & 16 & 33 & 43 & 60 & 56 & 4.51 & 1.255 \\
\hline Tareas individuales & 0 & 4 & 3 & 28 & 74 & 99 & 5.25 & 0.833 \\
\hline Trabajos en equipo & 9 & 13 & 34 & 51 & 69 & 32 & 4.22 & 1.307 \\
\hline
\end{tabular}

Fuente: elaboración propia.

Ahondando sobre esta cuestión, se comprobó que los estudiantes que obtenían las máximas calificaciones (sobresaliente), preferían participar en pruebas evaluativas como las tareas individuales $(\bar{x}=5.42$; $\mathrm{p}=0.038 ; \mathrm{F}=0.235 ; \hat{\mathrm{g}}=0.38)$ o los exámenes orales $(\bar{x}=3.96 ; \mathrm{p}=0.034 ; \mathrm{F}=0.132 ; \hat{\mathrm{g}}=0.20)$. Por ramas de conocimiento, se constató que los estudiantes pertenecientes al área de ciencias alcanzaron mejores resultados en exámenes de preguntas de desarrollo $\left(\bar{x}=5.19 ; \mathrm{p}=0.001 ; \mathrm{F}=5.069 ; \eta^{2}=0.091\right)$, los que cursaban estudios relacionados con ciencias de la salud lo hacían en las pruebas tipo test $(\bar{x}=4.86 ; \mathrm{p} \geq 0.000$; $\left.\mathrm{F}=9.648 ; \eta^{2}=0.160\right)$, los que estaban realizando una titulación vinculada al arte tenían mayor éxito en los exámenes orales $(\overline{\boldsymbol{x}}=4.22 ; \mathrm{p}=0.009 ; \mathrm{F}=3.474$; $\left.\eta^{2}=0.064\right)$ y, finalmente, el grupo de alumnos que se encontraba estudiando alguna ingeniería conseguía mejores calificaciones en las pruebas evaluativas de resolución de casos prácticos $(\bar{x}=5.13 ; \mathrm{p}=0.042$; $\left.\mathrm{F}=2.525 \eta^{2}=0.047\right)$.

Los estudiantes universitarios encuestados señalaron algunas claves que consideraron básicas para poder tener éxito en la enseñanza universitaria (tabla 5). Concretamente, indicaron que era importante terminar los trabajos y tareas de clase en las fechas establecidas, asistir a clase de manera regular, hacer resúmenes y realizar anotaciones en el texto mientras estudiaban, repasar los temas estudiados, planificar los tiempos de estudio y priorizar la formación ante cualquier otro tipo de actividad. Uno de los encuestados indicó que $\left(\mathrm{p}^{2}\right)$ "no me gusta escribir solo la idea que me da el profesor, sino coger los apuntes y hacer pequeños resúmenes, así al ir escribiendo los resúmenes también memorizas".

Los análisis de contraste realizados reflejaron que los estudiantes pertenecientes a la rama de ciencias sociales planteaban sus dudas al profesorado durante las explicaciones que realizaba en el aula $(\bar{x}=5.04$; $\left.\mathrm{p} \geq 0.000 ; \mathrm{F}=8.026 ; \eta^{2}=0.137\right)$, tenían en cuenta las recomendaciones del profesorado a la hora de enfrentar el proceso de aprendizaje $(\bar{x}=5.02 ; \mathrm{p}=0.044$; $\left.\mathrm{F}=2.496 ; \eta^{2}=0.047\right)$ y como principal estrategia de estudio, utilizaban el subrayado de texto para resaltar las ideas principales $(\bar{x}=5.34 ; \mathrm{p}=0.004 ; \mathrm{F}=3.978$; $\left.\eta^{2}=0.073\right)$. Por su parte, los estudiantes que cursaban estudios universitarios relacionados con el arte, llevaban al día los contenidos de las asignaturas $\left(\bar{x}=4.65 ; \mathrm{p}=0.006 ; \mathrm{F}=3.694 ; \eta^{2}=0.068\right)$ y utilizaban la biblioteca para consultar información $(\bar{x}=4.92$; $\mathrm{p} \geq 0.000 ; \mathrm{F}=9.147 ; \eta^{2}=0.153$ ). 
Tabla 5. Claves para tener éxito académico en la universidad

\begin{tabular}{|c|c|c|c|c|c|c|c|c|}
\hline & 1 & 2 & 3 & 4 & 5 & 6 & $\bar{x}$ & $d t$ \\
\hline Asistes a clase diariamente & 2 & 3 & 2 & 3 & 19 & 178 & 5.74 & 0.805 \\
\hline Planteas tus dudas al profesorado durante sus explicaciones & 9 & 26 & 24 & 32 & 53 & 63 & 4.37 & 1.536 \\
\hline Tienes en cuenta las recomendaciones del profesorado a la hora de estudiar & 4 & 4 & 25 & 44 & 61 & 69 & 4.74 & 1.206 \\
\hline Realizas una lectura previa al comenzar a estudiar un tema & 27 & 28 & 21 & 27 & 39 & 65 & 4.05 & 1.809 \\
\hline Realizas anotaciones en el texto cuando estudias & 9 & 9 & 4 & 20 & 45 & 120 & 5.14 & 1.353 \\
\hline Subrayas el texto para resaltar las ideas más importantes & 17 & 9 & 10 & 18 & 33 & 120 & 4.94 & 1.604 \\
\hline Haces esquemas y/o resúmenes de los temas & 16 & 13 & 15 & 29 & 35 & 99 & 4.70 & 1.622 \\
\hline Repasas los temas estudiados & 4 & 6 & 12 & 18 & 46 & 121 & 5.22 & 1.197 \\
\hline Estudias de memoria & 26 & 44 & 45 & 42 & 28 & 22 & 3.33 & 1.523 \\
\hline $\begin{array}{l}\text { Buscas por tu cuenta documentación complementaria a los apuntes de clase } \\
\text { para estudiar }\end{array}$ & 7 & 24 & 38 & 46 & 46 & 46 & 4.15 & 1.432 \\
\hline Llevas los contenidos de las asignaturas al día & 9 & 13 & 39 & 46 & 52 & 48 & 4.27 & 1.395 \\
\hline Realizas actividades de formación complementarias (cursos, jornadas, etcérera) & 44 & 42 & 39 & 28 & 32 & 22 & 3.14 & 1.661 \\
\hline Organizas y planificas el tiempo de estudio & 7 & 8 & 13 & 35 & 63 & 81 & 4.85 & 1.298 \\
\hline Terminas los trabajos y tareas de clase en las fechas establecidas & 0 & 0 & 2 & 9 & 30 & 166 & 5.74 & 0.583 \\
\hline Utilizas la biblioteca para consultar y buscar información & 14 & 24 & 24 & 29 & 56 & 60 & 4.30 & 1.594 \\
\hline Priorizas el estudio ante cualquier otra actividad & 0 & 11 & 22 & 39 & 47 & 88 & 4.86 & 1.227 \\
\hline
\end{tabular}

Fuente: elaboración propia.

\section{Competencias de adaptabilidad a los estudios universitarios}

Un objetivo básico de este estudio era determinar qué competencias tenían desarrolladas los estudiantes universitarios de éxito, para integrarse y adaptarse a la educación superior. Respecto a los procesos de transición y adaptación a los estudios universitarios, el alumnado valoró altamente la importancia que tenía la capacidad de tomar decisiones, tomar una decisión vocacional. Señalaba un estudiante que (p4) "lo primero es elegir bien. Si empiezas en la universidad a estudiar algo que sabes que no te gusta, no vas a estar motivado”. El alumnado destacó la importancia de elegir con criterio, con una buena información, de manera reflexiva y personal, para reducir el riesgo a equivocarse. Uno de los estudiantes encuestados manifestó que (p184) "es de vital importancia informarse sobre los grados que despierten interés. Muchas veces se accede a una carrera por probar suerte, lo cual es un grave error. Recomiendo analizar las asignaturas del grado antes de matricularse pues, aunque pueda parecer obvio, 
muchos ignoran en qué consiste el grado en cuestión, en que se han matriculado". Los resultados de esta investigación reflejaron que el 95.7\% de los encuestados tenía la percepción de tener desarrolladas las competencias básicas para superar la titulación que estaban cursando. Como se observa en la tabla 6, entendían que el éxito en su trayectoria académica universitaria se debía a que habían asumido la responsabilidad requerida para sacar adelante una titulación de grado, que tenían capacidad para resolver problemas, que sabían trabajar en equipo o que tenían habilidad para planificar y fijarse metas académicas y profesionales.

Tabla 6. Competencias básicas para afrontar con éxito la formación universitaria

\begin{tabular}{|c|c|c|c|c|c|c|c|c|}
\hline & 1 & 2 & 3 & 4 & 5 & 6 & $\bar{x}$ & $d t$ \\
\hline Te sientes capacitado para planificar y fijar metas académicas y profesionales & 4 & 5 & 11 & 46 & 84 & 58 & 4.80 & 1.097 \\
\hline Te sientes capacitado para expresarte y comunicarte correctamente & 1 & 6 & 20 & 56 & 80 & 45 & 4.65 & 1.043 \\
\hline Asumes la responsabilidad necesaria para sacar adelante la carrera que cursas & 1 & 0 & 3 & 13 & 70 & 121 & 5.47 & 0.748 \\
\hline Te sientes capacitado para trabajar en equipo con otros compañeros & 2 & 3 & 14 & 40 & 70 & 79 & 4.97 & 1.063 \\
\hline Te sientes capacitado para interactuar y relacionarte con otras personas & 2 & 4 & 13 & 31 & 77 & 80 & 5.01 & 1.059 \\
\hline $\begin{array}{l}\text { Te sientes capacitado para afrontar las decisiones que tienes que tomar y } \\
\text { resolver los problemas a los que te enfrentas }\end{array}$ & 1 & 1 & 4 & 42 & 88 & 72 & 5.07 & 0.862 \\
\hline Te sientes capacitado para el uso de los recursos tecnológicos & 2 & 6 & 16 & 39 & 76 & 69 & 4.87 & 1.108 \\
\hline Te consideras una persona creativa y con capacidad para generar buenas ideas & 2 & 3 & 28 & 47 & 70 & 58 & 4.70 & 1.115 \\
\hline Te consideras una persona con iniciativa y con capacidad emprendedora & 4 & 11 & 22 & 60 & 52 & 59 & 4.55 & 1.258 \\
\hline
\end{tabular}

Fuente: elaboración propia.

Estas competencias, que tienen un carácter genérico, no sólo se consideran relevantes para la vida académica, sino también para la futura vida laboral. Otro factor destacado para adaptarse y superar los estudios universitarios fue la constancia. En las preguntas abiertas, un estudiante señaló que (p1) "la constancia es imprescindible en la universidad. No creo que nadie saque una carrera bien si no está todos los días trabajando, aunque trabajar no implica solamente estudiar, sino estar atento en las clases, coger los apuntes, etcétera. La participación activa también implica involucrarse en los estudios y en la carrera, participar en las clases [...] Y después, algo que me ha ayudado bastante es la capacidad de relacionar. Creo que es importante entender los conocimientos que adquirimos en determinadas materias y que podemos aplicar a otras circunstancias".

Otro aspecto que se destacó, en relación con la adaptabilidad, fueron las expectativas positivas a la hora de afrontar el proceso de aprendizaje y la evaluación. Uno de los estudiantes puntualizó que (p2) "cuando hay un examen no se puede pensar en ir a conseguir un 5 de nota. Hay que pensar: yo voy a por el 10. Eso de ir a ver si apruebo, no; hay que ir a por la máxima nota. Si luego en vez de un 10 logras un 9, no pasa nada. Pero hay que estudiar y dedicar tiempo y esfuerzo".

El alumnado también valoró otras competencias para tener éxito en la universidad, como la reflexión, el manejo de los recursos virtuales o la gestión de la información. Al respecto, un estudiante planteó que (p2) 
"es fundamental la capacidad de reflexión [...] Otro aspecto que me ayudó bastante fue conocer y poner en práctica las nuevas tecnologías, no sólo en las prácticas educativas en los colegios, sino en el trabajo diario, en los trabajos que nos mandan los profesores: buscar, consultar distintas fuentes de información y esa información organizarla de tal forma que se pueda entender. En todos los trabajos que hago en la universidad no voy a lo fácil, a los apuntes y ya está, me gusta ir a la biblioteca, consultar un libro, buscar en internet todos los aspectos que tengan que ver con el trabajo".
Por otra parte, la escala utilizada para valorar el dominio de las competencias y tareas de adaptabilidad de los estudiantes universitarios de éxito (tabla 7), reveló que tenían desarrolladas diferentes habilidades relacionadas con este constructo. Concretamente, los datos recogidos indicaron que se trataba de personas que asumían la responsabilidad de sus propias acciones, superaban los obstáculos y dificultades que iban encontrando en su trayectoria personal y tenían claro que las decisiones tomadas podían influir en su futuro.

Tabla 7. Competencias y tareas de adaptabilidad

\begin{tabular}{|c|c|c|c|c|c|c|c|c|}
\hline & 1 & 2 & 3 & 4 & 5 & 6 & $\bar{x}$ & $d t$ \\
\hline Piensa en cómo puede ser su futuro académico y profesional & 2 & 5 & 7 & 24 & 72 & 97 & 5.17 & 1.033 \\
\hline Sabe que las decisiones de hoy influirán en su futuro académico y profesional & 2 & 1 & 4 & 17 & 63 & 120 & 5.41 & 0.881 \\
\hline Planifica cómo lograr sus metas y objetivos & 4 & 3 & 12 & 45 & 66 & 77 & 4.92 & 1.123 \\
\hline Es consecuente con sus metas y objetivos & 2 & 4 & 6 & 31 & 84 & 80 & 5.08 & 0.989 \\
\hline Es constante en el mantenimiento de sus planes & 1 & 4 & 9 & 50 & 78 & 65 & 4.91 & 0.988 \\
\hline Asume las responsabilidades de sus propias acciones y decisiones & 0 & 1 & 1 & 18 & 70 & 117 & 5.45 & 0.715 \\
\hline $\begin{array}{l}\text { Busca continuamente oportunidades para mejorar su desarrollo académico y } \\
\text { profesional }\end{array}$ & 0 & 9 & 29 & 45 & 62 & 62 & 4.67 & 1.169 \\
\hline Está motivada por seguir adelante a pesar de las dificultades & 1 & 2 & 7 & 28 & 69 & 100 & 5.23 & 0.932 \\
\hline Toma la iniciativa en el desarrollo de sus proyectos & 1 & 2 & 15 & 37 & 73 & 79 & 5.01 & 1.010 \\
\hline Piensa en las cosas que no hizo bien cuando no obtiene los resultados deseados & 1 & 1 & 7 & 17 & 63 & 118 & 5.39 & 0.873 \\
\hline Supera los obstáculos que va encontrando & 0 & 0 & 4 & 33 & 83 & 87 & 5.22 & 0.781 \\
\hline Se adapta con facilidad a los nuevos contextos & 1 & 2 & 20 & 53 & 59 & 72 & 4.85 & 1.067 \\
\hline Cree en sus posibilidades personales para tener éxito & 3 & 2 & 22 & 30 & 62 & 88 & 4.98 & 1.149 \\
\hline
\end{tabular}

Fuente: elaboración propia. 


\section{Proyecto formativo y expectativas profesionales}

Respecto al desarrollo profesional, el 63.9\% del alumnado señaló que tenía clara la profesión que le gustaría desempeñar en el futuro. La información aportada por los participantes, sugirió que los estudiantes universitarios de éxito tenían un adecuado conocimiento de las salidas profesionales vinculadas a los estudios que cursaban. En este sentido, al referirse a su futuro desarrollo profesional, algunos indicaron que (p4) "tengo tres alternativas por orden de prioridad: banca, asesoría fiscal y política macroeconómica/monetaria", (p87) "quiero trabajar en el campo del arte, específicamente en la gestión y conservación del patrimonio cultural y artístico, tanto en el campo de la investigación (que es la prioridad), como en el ámbito museístico" o (p141) "me gustaría ser doctora en Física y dedicarme a la investigación".

De los resultados obtenidos se desprendió que los participantes tenían una aspiración profesional bien definida, relacionada con un proyecto formativo y profesional que habían ido elaborando durante los estudios de grado y que les podría ayudar a transitar de la universidad al mercado de trabajo o, en algunos casos, a continuar cursando otros estudios de posgrado y de especialización. Algunos estudiantes incluso describieron de manera precisa el itinerario formativo y profesional que les podría llevar al mundo laboral al terminar los estudios de grado. $\mathrm{Al}$ respecto un alumno indició que iba a (p6) "realizar MIR $\rightarrow$ Escoger Anatomía Patológica $\rightarrow$ Ser Residente de Anatomía Patológica $\rightarrow$ Ser Patólogo $\rightarrow$ Docencia e Investigación".

El 36.1\% restante, aunque no tenían una idea precisa de la profesión que les gustaría desempeñar en su futuro, poseían información sobre las diferentes salidas profesionales de los estudios que cursaban. Concretamente, indicaron que (p2) "no sé a qué rama de la medicina dedicarme específicamente", (p8) "la carrera que curso tiene un amplio abanico de profesiones que desarrollar", (p98) "estoy dudando entre dos tipos de salida profesional que puede ofertar mis estudios" o (p137) "tengo varias opciones en mente, por un lado, salidas profesionales propias del Periodismo (trabajar escribiendo para algún medio de comunicación) y me interesa también el mundo académico y teórico".

\section{Conclusiones}

La principal conclusión a la que hemos llegado en este artículo es que para tener éxito en los estudios universitarios el alumnado debe tener una buena disposición hacia el aprendizaje, metas claras, responsabilidad respecto al oficio de estudiante y competencias adecuadas para afrontar con éxito el proceso formativo. Esta conclusión nos lleva a plantear que no es una sola variable la que influye y determina el buen rendimiento y éxito en los estudios, sino un conjunto de factores que intervienen de forma interrelacionada. En este sentido, coincidimos con los resultados de estudios como los de Garbanzo (2007), Ocaña (2011) o Mora (2015) donde también se identificaron factores de tipo social, cognitivo y emocional que tenían una fuerte vinculación con el rendimiento académico.

Los resultados académicos obtenidos en las etapas previas figuran como un predictor importante para lograr una buena adaptación a los estudios universitarios. En este caso, cabe destacar que el 95\% de los estudiantes que participaron en la investigación obtuvieron una nota media superior a notable en los estudios de bachillerato. Este dato evidencia que muchos estudiantes, desde las etapas previas, planifican su acceso a los estudios universitarios, se preocupen de obtener buenas calificaciones para poder acceder a la titulación que les interesa cursar y tienen un proyecto formativo y profesional bien definido. Las expectativas favorables de resultados, la motivación y el compromiso de los estudiantes con sus intereses son prerrequisitos fundamentales para el éxito académico. Por el contrario, como señalan Tejedor y GarcíaValcárcel (2007), aquellos estudiantes que afrontan el 
ingreso a una titulación universitaria sin la preparación, sin los conocimientos, sin motivación y sin aptitudes específicas acordes con las exigencias que les van a demandar, tendrán dificultades para integrarse y conseguir sus metas académico-profesionales.

La motivación por los estudios se relaciona directamente con la asistencia a clase, una de las variables académicas que los estudiantes de esta investigación señalaron como relevantes, puesto que les permite hacer un seguimiento de los temas tratados por el profesorado, comprender los conceptos básicos, aplicar estrategias de regulación del aprendizaje, estudiar con mayor confianza, etcétera. En los estudios de Montero y Villalobos (2004), Bartual y Poblet (2009) y Cueto y Rubiera (2013), se resalta la importancia que la asistencia a clase tiene en el proceso de aprendizaje del alumnado, ya que se considera una condición básica para hacer un buen seguimiento de las asignaturas y tener éxito en los estudios.

De los datos obtenidos se desprende que los estudiantes de éxito tienen un gran compromiso consigo mismos y con su proyecto formativo y profesional, priorizando sus metas a corto, medio y largo plazo. Muchos estudiantes no llegan a asumir que ser estudiante universitario supone algo más que dejarse llevar por la inercia que los conduce a matricularse en una titulación, la que sea, y sentarse en un pupitre frente a la tarima a escuchar lo que le dicen, sin cuestionarse nada, sin ninguna expectativa. Por eso se dice que a muchos estudiantes les falta "oficio" y el manejo de las competencias que ello conlleva. Un estudiante con oficio es aquel que valora lo que hace, piensa en su futuro, planifica sus estudios, lee y estudia regularmente, asiste y lleva al día sus clases, pregunta, resuelve dudas, repasa, relaciona e integra los aprendizajes en un proyecto formativo, tiene aspiraciones profesionales claras y se compromete con ellas.

En este sentido, coincidimos con Pérez, Quijano y Muñoz (2018) cuando comentan que el éxito en los estudios universitarios está condicionado por la dificultad para establecer una buena comunicación fluida con el profesorado, organizarse adecuadamente durante el tiempo en que el estudiante lleva a cabo el trabajo autónomo, entender las explicaciones y la terminología empleada por los docentes o saber utilizar los recursos de que dispone la universidad. El problema de la deserción académica se tiene que abordar de manera contextual, desde cada uno de los centros universitarios y con acciones prácticas que permitan, desde el momento en el que el alumnado accede a la universidad, facilitar y reforzar la integración social y académica, para lograr un adecuado compromiso con los estudios, motivación por la carrera y habilidades de tomar decisiones y hacer frente a los obstáculos, de modo que al final puedan alcanzar el éxito y lograr sus objetivos. Mayor inversión, mejores políticas y programas de refuerzo y apoyo al alumnado en su proceso de adaptación, mejora en los procesos de formación y sensibilización del profesorado, son algunos de los retos planteados para mejorar la calidad de la enseñanza universitaria y conseguir mayor éxito en los estudios.

Para finalizar, cabe señalar que, a través del estudio que hemos realizado, se ha podido definir el perfil de un estudiante capaz de obtener éxito en los estudios universitarios. Sería aquel que tiene un buen rendimiento académico en el bachillerato; tiene claro los estudios que quiere cursar y se preocupa por obtener buenas calificaciones en bachillerato para obtener plaza en la formación universitaria; tiene información precisa de los estudios que va a cursar y del perfil profesional de la titulación; conoce los requisitos de acceso a los estudios universitarios; valora la relación entre sus capacidades personales y las exigencias de los estudios; valora las consecuencias de las decisiones a tomar y elige después de tener en cuenta la diversidad de factores; está motivado por los estudios que cursa; estudia todos los días; asiste con regularidad a clase con el fin de familiarizarse con el contenido antes de estudiar y beneficiarse de las explicaciones que da el profesorado; domina las competencias genéricas básicas; planifica el proceso 
de estudio; aplica estrategias para rentabilizar el aprendizaje; prioriza el estudio sobre otras actividades; tiene claro qué profesión le gustaría desempeñar en el futuro y se prepara para ello.

Los resultados de la investigación realizada no son extrapolables al conjunto de universidades españolas, puesto que corresponden de manera exclusiva a la Universidad de La Laguna. No obstante, constituyen

\section{Referencias}

Álvarez, M., P. Figuera y M. Torrado (2011), "La problemática de la transición bachillerato-universidad en la Universidad de Barcelona", Revista Española de Orientación Psicopedagógica, 22 (1), pp. 15-27.

Álvarez, P. R., D. López y D. Pérez (2015), “Análisis de los factores que intervienen en la transición del bachillerato a la universidad", en AIDIPE (Asociación Interuniversitaria de Investigación en Pedagogía) (Ed.), Investigar con y para la sociedad, Madrid, Bubok Publisihing, pp. 599-608.

Álvarez, P., L. Cabrera, M. González y J. Bethencourt (2006), "Causas del abandono y prolongación de los estudios universitarios", Paradigma, 27 (1), pp. 7-36.

Álvarez, P. y D. López (2017), "Recursos de Orientación para la transición académica y la toma de decisiones del alumnado: el Programa 'Universitarios por un día"', Revista d'Innovació Docent Universitària, 9, pp. 26-38.

Bartual, T. y C. Poblet, (2009), "Determinantes del rendimiento académico en estudiantes universitarios de primer año de Economía", Revista de Formación e Innovación Educativa Universitaria, 2 (3), pp. 172-181.

Belvis, E., M. Moreno y F. Ferrer (2009), "Los factores explicativos del éxito y fracaso académico en las universidades españolas, en los años del cambio hacia la convergencia europea", Revista española de educación comparada, 15, pp. 61-92.

Bethencourt, J., L. Cabrera, J. Hernández, P. Álvarez y M. González (2008), "Variables psicológicas y educativas un punto de partida para profundizar en los factores asociados al éxito académico y la planificación de intervenciones de apoyo al alumnado. Asimismo, estos resultados pueden orientar investigaciones centradas en el análisis de otras variables asociadas al éxito en los estudios, que resulten de interés para disminuir el efecto de factores que provocan el fracaso y abandono de los estudios.

en el abandono universitario", Revista Electrónica de Investigación Psicoeducativa, 18, pp. 603-622.

Cabrera, L., J. Bethencourt, P. Álvarez y M. González (2006), "El problema del abandono de los estudios universitarios", Revista Electrónica de Investigación y Evaluación Educativa, 12 (2), pp. 107-203.

Castaño, E., S. Gallón, K. Gómez y J. Vázquez (2008), "Análisis de los factores asociados a la deserción estudiantil en la Educación Superior", Revista de Educación, 345, pp. 255-280.

Coe, R. y C. Merino (2003), "Magnitud del efecto: una guía para investigadores y usuarios", Revista de Psicología de la PUCP, XXI (1), pp. 146-177.

Cohen, J. (1969), Statistical power analysis for the behavioral Sciences, Nueva York, Academic Press.

Cueto, B. y F. Rubiera (2013), "El éxito académico en la adaptación de la asignatura Economía Española y Europea al EEES. Reflexiones a partir de la experiencia en la Facultad de Economía y Empresa de la Universidad de Oviedo", Revista de Docencia Universitaria, 11 (2), pp. 263-285.

Elías, M. (2008), "Los abandonos universitarios: retos ante el espacio europeo de educación superior", Estudios sobre Educación, 15, pp. 101-121.

Etxeberría, J. y F. J. Tejedor (2005), Análisis descriptivo de datos en educación, Madrid, La Muralla.

Feldman, R. S. (2005), Improving the first year of college: Research and practice, Mahwah, NJ, LEA. 
Garbanzo, G. (2007), "Factores asociados al rendimiento académico en estudiantes universitarios, una reflexión desde la calidad de la educación superior pública", Revista Educación, 31 (1), pp. 43-63.

García, A. (2014), "Rendimiento académico y abandono universitario: Modelos, resultados y alcances de la producción académica en la Argentina", Revista Argentina de Educación Superior, 8, pp. 9-38.

García, M., A. Bernardo y L. Rodríguez (2016), "Permanencia en la universidad: la importancia de un buen comienzo", Aula abierta, 44 (1), pp. 1-6.

García-Aretio, L. (2019), "El problema del abandono en estudios a distancia. Respuestas desde el diálogo didáctico mediado", Revista Iberoamericana de Educación a Distancia, 22 (1), pp. 245-270.

George, D. y P. Mallery (2003), SPSS for Windows step by step: a simple guide and referent. 11.0 Update, Boston, Allyn \& Bacon.

González, M., P. Álvarez, L. Cabrera y J. Bethencourt (2007), "El abandono de los estudios universitarios: factores determinantes y medidas preventivas", Revista Española de Pedagogía, LXV (236), pp. 71-85.

Gonzalo, F. y F. García (2016), "Permanencia y abandono de estudios en estudiantes universitarios: un análisis desde la teoría organizacional", Revista de la Educación Superior, 45 (179), pp. 25-39.

Granada, H. (2003), "La cultura como estrategia de adaptación en la interacción sujeto social-ambiente", Investigación y Desarrollo, 11 (1), pp. 134-161.

Himmel, E. (2018), "Modelo de análisis de la deserción estudiantil en la educación superior", Calidad en la Educación, 17, pp. 91-108.

Honike, T. y J. Broadbent (2016), "The influence of academic self-efficacy on academic performance: A systematic review", Educational Research Review, 17, pp. 63-84.

Jansen, E. y C. Shure (2010), "The effect of secondary school study skills preparation on first-year university achievement", Educational Studies, 36 (5), pp. 569-580.

Lent, R., G. Hackett y S. Brown (2004), "Una perspectiva social cognitiva de la transición entre la escuela y el trabajo", Evaluar, 4, pp. 1-22.

Lent, R., M. Taveira, H. Sheu y D. Singley (2009), "Social cognitive predictors of academic adjustment and life satisfaction in Portuguese College students: a longitudinal analysis", fournal of Vocational Behavior, 74 (2), pp. 190-198.

Lent, R. y G. Hackett, (1987), "Career self-efficacy: empirical status and future directions", fournal of Vocational Behavior, 30, pp. 347-382.

López, F. (2002), "El análisis de contenido como método de investigación”, XXI, Revista de Educación, 4, pp. 167179.

Martín, E., M. González, J. Rodríguez, C. Pérez, P. Álvarez, L. Cabrera, J. Bethecourt, C. Páez y A. Palenzuela (2010), El rendimiento académico del alumnado de nuevo ingreso en la Universidad de La Laguna, La Laguna, Servicio de Publicaciones de la Universidad de La Laguna.

McMillan, E. y S. Schumacher (2005), Investigación Educativa (5 ${ }^{\text {a }}$ Edición), Madrid, Pearson.

Montero, E. y J. Villalobos (2004), Factores institucionales, pedagógicos, psicosociales y sociodemográficos asociados al rendimiento académico y a la repetición estudiantil en la Universidad de Costa Rica, Costa Rica, Instituto de Investigaciones Psicológicas, Universidad de Costa Rica.

Mora, R. (2015), "Factores que intervienen en el rendimiento académico universitario: Un estudio de caso", Opción, 6, pp. 1041-1063.

Nora, A. y G. Crips (2012), "Student persistence and degree attainment beyond the first year in college: Exixting knowledge and directions for future research", en A. Seidman (ed), College student retention. Formula for student success, Rowman y Littlefield publishers.

Ocaña, Y. (2011), "Variables académicas que influyen en el rendimiento académico de los estudiantes universitarios", Investigación Educativa, 15 (27), pp. 165-179.

Pascarella, E.T. y P.T. Terenzini (1991), How college affects students: findings and insights from twenty years of research, San Francisco, Jossey Bass.

Pérez, M., R. Quijano y I. Muñoz (2018), "Transición de secundaria a la universidad en estudiantes de los 
títulos de maestro de Educación Infantil y Primaria de la Universidad de Jaén", Aula Abierta, 47 (2), pp. 167 176.

Ramírez, M. I., F. Herrera y I. Herrera (2003), “¿Qué ocurre con la adaptación y el rendimiento académico de los alumnos, en un contexto educativo pluricultural?", Revista Iberoamericana de Educación, 33 (2), pp. 1-17.

Rodríguez, S., E. Fita y M. Torrado (2004), "El rendimiento académico en la transición secundariauniversidad", Revista de Educación, 334, pp. 391-414.

Rué, J. (2013), "El abandono universitario: variables, marcos de referencia y políticas de calidad", REDU: Revista de Docencia Universitaria, 12 (2), pp. 281-306.

Sánchez, A. y M. Elías (2017), "Los estudiantes universitarios no tradicionales y el abandono de los estudios", Estudios sobre Educación, 32, pp. 27-48.

Savickas, M. (2012), "Life design: a paradigm for career intervention in the 21 st Century", fournal of Counseling E Development, 90, pp. 13-19.

Sousa, R., A. Lopes y E. Ferreira (2013), "La transición y el proceso de adaptación en la educación superior: un estudio con estudiantes de una escuela de enfermería y de una escuela de educación", Revista de Docencia Universitaria, 11 (3), pp. 403-422.

Tejedor, F. J. y A. García-Valcárcel (2007), “Causas del bajo rendimiento del estudiante universitario (en opinión de los profesores y alumnos). Propuestas de mejora en el marco del EEES", Revista de Educación, 342, pp. 443-473.

Tinto, V. J. (2006), "Research and practice of student retention: what next?", College Student Retention, 8 (1), pp. 1-19.

Villar, A. (2010), "Del abandono de estudios a la reubicación universitaria", Revista de la Asociación de Sociología de la Educación, 3 (2), pp. 267-283.

Yokoyama, S. (2018), "Academic Self-Efficacy and Academic Performance in Online Learning: A Mini Review", Front Psychology, 9, pp. 2794.

Zepke, N. y L. Leach (2010), "Improving stude nt engagement: ten proposals for action", Active Learning in Higher Education, 11 (3), pp. 167-177.

\section{Cómo citar este artículo:}

Álvarez-Pérez, Pedro-Ricardo y David López-Aguilar (2020), "Competencias de adaptabilidad y factores de éxito académico del alumnado universitario", Revista Iberoamericana de Educación Superior (RIES), vol. XI, núm. 32, pp. 46-66, DoI: https://doi.org/10.22201/ iisue.20072872e.2020.32.815 [Consulta: fecha de última consulta]. 